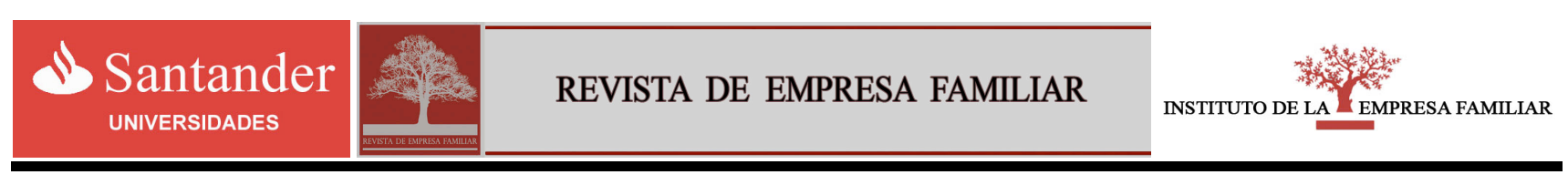

www.revistadeempresafamiliar.uma.es

\title{
Importancia del concepto de Empresa Familiar en investigación: utilización de la base de datos SABI para su clasificación
}

\section{Importance of the concept of family business in research: the use of 'SABI' database for its classification}

\author{
Alfonso A. Rojo Ramírez ${ }^{\mathrm{a}, *} \cdot J^{*}$ Julio Diéguez Soto ${ }^{\mathrm{b}} \cdot$ Pilar López Delgado $^{\mathrm{c}}$ \\ ${ }^{\mathrm{a}}$ Departamento de Dirección y Gestión de Empresas. Universidad de Almería, 04120 (Spain) \\ ${ }^{\mathrm{b}}$ Departamento de Finanzas y Contabilidad. Universidad de Málaga, 29071 (Spain) \\ ${ }^{\mathrm{c}}$ Departamento de Estadística y Econometría. Universidad de Málaga, 29071 (Spain)
}

D A T O S A R T Í C U L O

Historial:

Recibido 08-07-2010

Aceptado 18-02-2011

Palabras clave:

Empresa familiar

Bases de datos

SABI

Tipología de empresas

Códigos JEL:

M10, M14, M20, M21, M40

\footnotetext{
A R T I C LE IN F O

Article history:

Received 08 july 2010

Accepted 18 february 2011
}

Keywords:

Family business

Databases

SABI

Business typology

JEL codes:

M10, M14, M20, M21, M40

\section{R E S U M EN}

Este trabajo tiene que ver con aspectos conceptuales de la empresa familiar (EF) y su impacto en la investigación. Su objetivo es servir de guía en el desarrollo de trabajos empíricos que pretenden disociar la EF de la empresa no familiar (EnF) mediante la utilización de bases de datos, particularmente contables y, más concretamente, en el caso español, con la base SABI.

En él se pone de manifiesto la importancia, en el ámbito empírico, de buscar un mecanismo que nos permita identificar la EF de forma eficiente, así como las dificultades existentes para ello con el uso de SABI. Trabajar con diferentes criterios de selección y el uso de apellidos nos permite proponer una tipología de EF.

A B S T R A C T

This paper deals with conceptual aspects of the family business (FB) and its impact on research. Its aim to give guidance on the development of empirical research dissociating FB and non family business (nFB) through the use of databases, particularly accounting data and, more specifically, in the Spanish case, the SABI database.

It highlights the importance, in the empirical field, to seek a mechanism that allows us to identify the FB efficiently, as well as the difficulties in using SABI database. Working with different selection criteria and the use of surnames allows us to propose a typology of FB.

\footnotetext{
* Autor de contacto. Tel.: +34950246707.

Correoselectrónicos: arojo@ual.es,juliodieguezsoto@uma.es,dlp@uma.es
} 


\section{Introducción}

Gran parte de la investigación sobre empresa familiar (EF) trata de comprobar si ésta se comporta de forma diferente a la empresa no familiar $(E n \mathrm{~F})$, tal como hacen los trabajos de Chrisman, Chua y Sharma, (2003); LópezGracia y Sánchez-Andújar, (2007); Villalonga y Amit, (2006); García de la Borbolla, et al. (2007) o Allouche et al, (2008), entre otros.

Aunque hoy parece superado el debate acerca de si la EF debe ser estudiada de forma diferenciada a la EnF (Basco, 2006), dado el importante desarrollo que esta materia ha alcanzado desde finales del pasado siglo, parece necesario profundizar en la definición de EF por el impacto que puede tener en el campo de la investigación.

No es fácil llegar a un acuerdo sobre este asunto, tal como se ha subrayado en el ámbito económico (Ariza; Molina y Ramírez, 2005) y el jurídico (Rodríguez, 2006). Lo cierto es que existe una falta de definición universalmente aceptada para la empresa familiar (Astrachan y Shanker, 2003: 218).

El presente trabajo tiene como objetivo analizar el concepto de EF y, en particular, su impacto en la investigación empírica que se sustenta en bases de datos contables.

Para ello, el siguiente apartado aborda las diferentes perspectivas del concepto de EF y, en particular, el sustentado en aspectos cuantitativos. El apartado tercero plantea el concepto de EF en el ámbito empírico español.

El apartado cuarto trata de definir un concepto adecuado de empresa dentro de las posibilidades dadas por la base de datos de empresas SABI, estratificada por tamaño y sector, que permita diferenciar adecuadamente las EFs de las EnFs.

Por su parte, el apartado quinto establece un concepto operativo de EF que permite su discriminación de la EnF. El epígrafe sexto conecta el concepto teórico con el concepto operativo de EF. Por último, el apartado séptimo concluye.

\section{La preocupación por el concepto de empresa familiar}

Parece evidente que no resulta fácil definir la EF. Por ejemplo, en Europa se han identificado hasta 90 definiciones distintas (Mandl, 2008:20) que hacen hincapié en diferentes componentes, tales como la influencia sobre la propiedad y el control, la participación de la familia sobre la actividad empresarial y su gestión, la contribución de la empresa a la riqueza de los propietarios o consideraciones de tipo intergeneracional como es la transmisión de padres a hijos.

La falta de un consenso generalizado es un serio problema desde el punto de vista político, económico y social (Mandl, 2008:8), pues la falta de una definición admitida no permite adoptar medidas orientadas a influir en su comportamiento como tampoco avanzar en su conocimiento (Chrisman, Chua y Sharma, 2005:556).

A pesar de lo dificultoso que es poder delimitar EF y EnF (Tsang, 2002), se ha dedicado un importante esfuerzo (Handler, 1989:258; Sharma, 2004:4) en este sentido (por ejemplo1: Litz, 1995; Chua, Chrisman y Sharma, 1999; Habbershon, Williams y MacMillan, 2003) aunque, como señalan Chrisman, Chua y Sharma (2005:557), se hace necesario diferenciar entre un concepto teórico, útil para crear teoría (essence approach), y otro concepto operativo, centrado en los componentes que se ven implicados a la hora de clasificar a una empresa como familiar o no, (components-of-involvement approach).

Sobre la primera de las aproximaciones, cuya relevancia subraya Basco (2006: 40), son numerosos los autores que señalan el interés de ir más allá de los elementos meramente cuantitativos si deseamos ofrecer una definición adecuada de EF (Chrisman, Chua y Litz, 2003; Chrisman; Chua y Sharma, 2003).

Astrachan y Shanker (2003:211-212) crean un rango de definiciones de posibles empresas familiares, en función del grado percibido de

${ }^{1}$ Más de 30 definiciones de diferentes autores se pueden encontrar en el trabajo de Ariza, Molina y Ramírez (2005). 
implicación familiar en la empresa. Sin embargo, es evidente la dificultad de este acercamiento a la $\mathrm{EF}$, por lo complejo que es apreciar el diferente grado de implicación de la familia en la empresa (Sharma, P 2004:4). Así, basado en el estudio de 217 artículos estos autores llegan a diferenciar tres líneas de actuación en este campo: a) aquella que utiliza un concepto multioperativo que permite establecer diferentes tipos de EF; b) aquella que desarrolla escalas con el objetivo de capturar la influencia de la familia en la empresa; c) aquella que se centra en establecer diferentes tipologías de EF.

La segunda de las aproximaciones es la que más preocupa desde el punto de vista de la investigación empírica, pues lo que interesa es una definición operativa que permita efectuar la separación entre EF y EnF, para así poder apreciar los elementos que diferencian a unas de las otras y avanzar en el conocimiento de las mismas.

En este sentido, parte de la literatura existente, por mor de conceptualizar teóricamente de manera correcta la $\mathrm{EF}$, coincide en señalar (Comblé et Colot, 2006:91) que existen tres elementos que deben darse para clasificar una empresa como familiar:

1. El control del capital por la familia.

2. La participación activa de la familia en la gestión de la empresa.

3. La transmisión o la voluntad de transmitir la empresa a la generación siguiente.

Algunos autores utilizando este sistema multicriterio añaden componentes emocionales. Por ejemplo, proponen utilizar tres criterios básicos para identificar cuando una empresa es EF (Smith, 2007:11):

1. La percepción de EF por parte de los miembros de un grupo con parentesco emocional (emotional kinship group). Respondería a la siguiente pregunta: ¿Consideras que tu empresa es una EF?

2. Que la empresa esté gestionada por algún miembro de una unidad familiar. Respondería a la pregunta: ¿Consideras que la empresa es familiar porque los miembros de la familia son directores?
3. Cuando una familia posee más del $50 \%$ de las acciones de la empresa. Respondería a la pregunta: ¿Consideras que la empresa es familiar porque los propietarios detentan más del 50\% del capital?

Sin embargo, y tal y como señalan Astrachan y Shanker (2003:213) "las características utilizadas más a menudo para definir la empresa familiar son dificiles, sino imposibles, de recopilar".

Por ello, muchos autores tratan de hacer más operativa esta definición dejando exclusivamente factores cuantitativos en la misma (Allouche, et al, 2008:316; Anderson y Reeb, 2003; Villalonga y Amit, 2006; Chirico et al, 2007: Nota 3; Westhead y Cowling, 1999), limitándose a verificar la existencia de los criterios citados previamente.

Sobre la base de estos tres elementos, el European Group of Owner Managed and Family Enterprises (GEEF) aprobó el 27 de mayo de 2008 una definición genérica que incluye los siguientes elementos y que ha hecho suya el Instituto de la Empresa Familiar (IEF) con pequeñas variantes:

1. Que la familia pueda ejercer el control accionarial de la compañía, bien sea por poseer la mayoría de los votos, o por su posibilidad de ejercer una influencia decisiva sobre los aspectos fundamentales del gobierno corporativo.

2. Tener una presencia relevante en los órganos de gobierno corporativo de la compañía (habitualmente el Consejo de Administración).

3. La participación directa de un miembro de la familia en la gestión de la empresa a su máximo nivel.

\section{El concepto de empresa famliar y la investigación con empresas familiares en España}

Tal como acabamos de señalar, una de las preocupaciones principales a la hora de abordar el trabajo empírico con $\mathrm{EF}$, es definir adecuadamente el campo de estudio, la EF. En este sentido se hace necesario adoptar un concepto operativo, centrado en los componentes implicados a la hora de clasificar 
la empresa como familiar o no (components-ofinvolvement approach) (Chrisman, Chua $\mathrm{y}$ Sharma, 2005).

La dificultad para incorporar factores no cuantitativos (Sharma, P. 2004) ha hecho que numerosas investigaciones se decanten por estudios sustentados en encuesta (por ejemplo: Gallo, Tàpies y Cappuyns, 2004:303, Westhead y Howorth, 2006:306; Heck y Trent, 1999:212; García de la Borbolla et al, 2007:107; Westhead y Cowling, 1997:35; Smith, 2008:50), normalmente a través de un cuestionario dirigido al gerente-propietario de la empresa.

Este método tiene el inconveniente de que, salvo en las empresas pequeñas, las cuestiones de la encuesta no suelen ser contestadas por miembros de la familia, que son los que conocen su estructura última, sino por personal de la misma (secretarios,...) que no siempre están al corriente de tales aspectos.

Cuando no ha sido así, los investigadores han tomado sus datos de muestras sustentadas en bases de datos del mercado a las que han aplicado filtros basados en aspectos cuantitativos como los indicados en el apartado precedente (por ejemplo, para España: Monreal et al, 2009; García de la Borbolla, et al. 2007).

Como ejemplo paradigmático de la búsqueda de una definición operativa de EF podemos hacer referencia a Villalonga y Amit (2006:390) quiénes consideran como tal aquélla cuyo fundador o un miembro de la familia de sangre, o por matrimonio, es un miembro del equipo de dirección, miembro del consejo de administración, o el propietario de al menos el $5 \%$ del capital de la empresa, individualmente o como un grupo. Sin embargo, en su trabajo, combinando los anteriores requisitos, toman hasta 9 diferentes definiciones de EF para comprobar su efecto en el valor de la empresa.

Al igual que el trabajo anterior, la mayoría de los trabajos de investigación, utilizan datos de empresas cotizadas, lo que restringe en gran medida el número de elementos que manejan. Toman como referencia la información suministrada para las empresas que cotizan en los mercados de valores, como los de la CNMV (Santana y Cabrera, 2001:10), aquéllos de las empresas del índice S\&P 500 (Lee, 2009:104; Anderson y Reeb, 2003:1308 ), del Main Board of Bursa Malaysia (Noor y Ayoib, 2009:15) o de la Bolsa de Comercio de Santiago (Martínez, Stohr y Quiroga, 2007: 83). Algunos se inclinan por las empresas más grandes, como, por ejemplo, la lista publicada de forma anual por la revista Fortune: Fortune 500.

Cuando se desea trabajar con EF no cotizadas en España, nos encontramos que no existen muchas fuentes de información disponibles e, incluso, la tendencia a trasladar trabajos empíricos de otros países puede resultar en un fracaso, pues tal como señala Allouche et al, (2008:315), la definición de EF suele variar según el país donde nos situemos, ya que el concepto de familia varía en las diferentes culturas.

Además, las características de las bases de datos disponibles no son homogéneas entre países.

Normalmente, a nivel internacional se utilizan bases de datos como COMPUSTAT DATA (McConaughy et al, 1998:4; McConaughy et al, 2001:6) o WORLDSCOPE DATABASE (Allouche et al, 2008:320), mientras que en España se suele utilizar la Base de datos SABI (Garcia de la Borbolla, et al. 2007 y 2006; López-Gracia, J. y SánchezAndújar, S. 2007) y hay que tener en cuenta que las particularidades de cada país requieren un estudio de las características de los datos utilizados antes de proceder a una traslación en el uso de la base de datos.

\section{Definición de empresa a partir de la base de datos $S A B I$ y su clasificación en sectores}

Gran parte de las investigaciones sobre EF precisan diferenciar entre $\mathrm{EF}$ y $\mathrm{EnF}$, como ocurre en los trabajos sobre eficiencia, solvencia o valoración de mercado.

En estos casos, si se precisa utilizar datos a obtener de la base SABI nos encontramos con el problema básico de que ésta no diferencia entre uno y otro tipo de empresas.

Es dramático observar cómo los resultados pueden llegar a conclusiones muy distintas 
según el concepto de EF elegido (Gestead y Cowling, 1998), máxime si la base de datos no se ha trabajado muy detenidamente.

Para poder identificar las EF dentro de SABI es necesario partir de un concepto adecuado de empresa que nos permita posteriormente poder comparar ambas tipologías y asegurarnos que las diferencias detectadas realmente obedecen a una diferencia real, y no a otros hechos que nada tienen que ver con el objeto de investigación.

La formación de una base de datos operativa para el estudio de la EF precisa de una estrategia de búsqueda que debería responder a los siguientes patrones:

1. Identificación de las empresas para las que existen datos disponibles, según el objeto de la investigación, durante todo el periodo considerado en la misma.

2. Selección de entre las anteriores, de aquéllas que estén activas, esto es, que no hayan comenzado un proceso concursal, ni hayan sido disueltas, absorbidas o extinguidas, o se encuentren inactivas.

3. Tratando de buscar EFs conviene despreciar aquellas formas jurídicas que no sean de tipo capitalista, por lo que hay que dejar fuera las cooperativas, las asociaciones y no definidas, por no encuadrarse dentro del ámbito familiar;

4. Por razones de actividad, y utilizando el CNAE 2009, generalmente se dejan fuera las empresas que, a nivel de dos dígitos, aparecen reflejadas en la tabla 1. Es decir, las empresas financieras (códigos 64, 65 y 66), ya que requieren un tratamiento diferenciado, y el resto porque sus datos contables suelen actuar como outliers, (por ejemplo, confederaciones de empresarios, organizaciones no lucrativas, $\mathrm{o}$ empresas municipales).

Así, bajo este criterio las empresas existentes en la base de datos SABI, a fecha 31 de diciembre de 2009 , que podrían ser objeto de estudio serían de 525.447.
Tabla 1

Sectores suprimidos del concepto de empresa.

\begin{tabular}{cl}
$\begin{array}{c}\text { Código } \\
\text { CNAE } \\
2009\end{array}$ & Descripción \\
\hline 64 & $\begin{array}{l}\text { Servicios financieros, excepto seguros y } \\
\text { fondos de pensiones }\end{array}$ \\
65 & $\begin{array}{l}\text { Seguros, reaseguros y fondos de pensiones, } \\
\text { excepto Seguridad Social obligatoria }\end{array}$ \\
66 & $\begin{array}{l}\text { Actividades auxiliares a los servicios } \\
\text { financieros y los seguros }\end{array}$ \\
84 & $\begin{array}{l}\text { Administración Pública y defensa; } \\
\text { Seguridad Social obligatoria }\end{array}$ \\
94 & $\begin{array}{l}\text { Actividades asociativas } \\
\text { Actividades de los hogares como } \\
\text { empleadores de personal doméstico }\end{array}$ \\
97 & $\begin{array}{l}\text { Actividades de los hogares como } \\
\text { productores de bienes y servicios para uso } \\
\text { propio } \\
\text { Actividades de organizaciones y organismos } \\
\text { extraterritoriales }\end{array}$ \\
Fuente: elaboración propia a partir de la Base de Datos SABI
\end{tabular}

Habitualmente se suelen evitar las empresas 'micro', entre otras circunstancias (Maseda et al, 2009): por tener una alta tasa de nacimientos y decesos; porque su grado de aportación al estudio suele estar muy limitado dado el peso económico de las mismas en el conjunto de la economía y porque su información tiende a ser muy poco precisa deteriorando la calidad de la información financiera del conjunto.

En el caso de que se optara por su exclusión se puede utilizar la recomendación de definición de tamaño empresarial de 2003/361/CE, de 6 de mayo de 2003, de la Comisión de las Comunidades Europeas. No obstante, la finalidad de la investigación puede aconsejar la inclusión de este tipo de empresas.

Por ejemplo, en un trabajo sobre PYMES familiares podría ser necesaria su inserción, aunque si el estudio exige la aplicación posterior de métodos estadísticos paramétricos podría dar lugar a una excesiva variabilidad de su análisis económico-financiero, lo que impediría el cumplimiento de las hipótesis de partida que exigen dichos métodos. En la tabla 3 se ve una simulación de ambos casos.

Algunos autores clasifican las empresas en estratos de tamaño, porque éste puede influir en los resultados de las variables objeto de medición (García de la Borbolla et al, 2007:112; McConaughy et al, 2001:6; Gardey et al, 
2008:p47; Westhead y Cowling, 1997:32; Smith,2008:50; Allouche et al, 2008:319). La justificación de esta forma de trabajar descansa en que se considera que si no se estratifica por distinto tamaño existe el peligro de que se observen diferencias entre EFs y EnFs, que no obedecen realmente a su carácter familiar, sino a su mayor presencia en determinados estratos empresariales.

Al trabajar con estratos de tamaño en SABI se ha de conocer el número de empleados, el total activo y el importe neto de la cifra de ventas para cada empresa.

Como no existen dichos datos para todas las empresas SABI, hay una reducción en el número de empresas disponibles ${ }^{2}$. A fecha 31 de diciembre de 2009 dicho número de empresas se reparte en diferentes tamaños según se explicita en la tabla 2.

\section{Tabla 2}

Empresas de la población por tamaño.

\begin{tabular}{ll}
\hline Tamaño & Número de empresas \\
\hline Micro & 295.175 \\
Pequeña & 93.690 \\
Mediana & 16.068 \\
Grande & 3.363 \\
\hline Total & $\mathbf{4 0 8 . 2 9 6}$ \\
\hline
\end{tabular}

Fuente: elaboración propia a partir de SABI

La tabla 3 muestra un ejemplo de estrategia de búsqueda a la hora de seleccionar la población objeto de estudio.

Los elementos de la población serían las empresas españolas cuya forma jurídica es una sociedad anónima, limitada, regular colectiva o comanditaria, para las que existen cuentas disponibles en todo el periodo 2002-2007, activas y que no son "microempresas". Son un total de 408.296 empresas si se incluyen las microempresas y 113.121, si no se consideran.

Dyer, (2006:254); García de la Borbolla, et al, (2007:107); McConaughy et al, (2001:6); Gardey et al, (2008:47); Westhead y Cowling (1997:35); Smith, (2008:10); Allouche et al,

${ }^{2}$ El número total de empresas por tamaño resulta inferior al total calculado sin esa variable.
(2008:319) dividen la muestra por sectores. El porqué de esta forma de actuar está en que, al igual que ocurría con el tamaño de la empresa, se teme que las diferencias observadas entre EF y EnF no sean debidas a su idiosincrasia sino a la mayor abundancia de empresas familiares en un determinado sector.

Se puede dividir la población siguiendo la clasificación de la Contabilidad Nacional del INE que distingue 4 grandes sectores: agricultura, industria, construcción y servicios. SABI permite, entre otras, la clasificación por sectores según el Impuesto de Actividades Económicas (IAE) ó según CNAE -en diferentes versiones: 1993, 2009,..-. Dado que a nivel internacional la clasificación del IAE no es un estándar, es preferible trabajar con la CNAE2009 que está coordinada con la europea $^{3}$.

La CNAE2009 ofrece un nivel máximo de agregación de dos dígitos, lo que permite trabajar con un amplio número de sectores.

Sin embargo, investigar con un gran nivel de desagregación sectorial dificulta el análisis de datos posterior, por la falta de suficiente número de observaciones (empresas) para cada subsector. Por ello, finalmente, se opta por la CNAE-2009 a solo 4 sectores (Ver tabla 4).

El número de empresas por sector y tamaño que ofrece SABI para el ejemplo anteriormente comentado ${ }^{4}$ se muestra en la tabla 5 .

\footnotetext{
${ }^{3}$ La comparabilidad internacional de las estadísticas producidas según la CNAE-2009 está garantizada por el hecho de formar parte de un sistema integrado de clasificaciones económicas de actividades y productos. El CNAE2009 es la versión nacional de la NACE Rev.2 en el ámbito de la Unión Europea.

${ }^{4}$ No son coincidentes la cifra total de empresas desagregadas por sector y tamaño (tabla 5) con el número de empresas resultantes de la estrategia de búsqueda (tabla 3). La diferencia obedece normalmente a que hay empresas clasificadas en más de un sector.
} 
Tabla 3

Estrategia de búsqueda de la población de empresas.

\begin{tabular}{|c|c|c|c|}
\hline Descripción & Detalles de búsqueda & $\begin{array}{l}\mathrm{N}^{0} \text { de empresas } \\
\text { (con } \\
\text { microempresas) }\end{array}$ & $\begin{array}{l}N^{0} \text { de empresas } \\
\text { (sin } \\
\text { microempresas) }\end{array}$ \\
\hline Años disponibles & Período 2002-2007 & 569.851 & 569.851 \\
\hline Estado & Activa & 543.154 & 543.154 \\
\hline País & España & 543.154 & 543.154 \\
\hline Forma jurídica & $\begin{array}{l}\text { Sociedad Anónima, Sociedad } \\
\text { Limitada, Sociedad Regular } \\
\text { colectiva, sociedad } \\
\text { Comanditaria }\end{array}$ & 540.657 & 540.657 \\
\hline Actividad & $\begin{array}{l}\text { Todos los códigos de CNAE } 09 \\
\text { a excepción de } 64,65,66,84, \\
94,97,98 \text { y } 99\end{array}$ & 525.447 & 525.447 \\
\hline $\begin{array}{l}\text { Número de } \\
\text { empleados y total } \\
\text { Activo o Importe } \\
\text { neto de Cifra de } \\
\text { Ventas }\end{array}$ & $\begin{array}{l}\text { Ver tabla } 2 \text { y recomendación } \\
2003 / 361 / \text { CE de la comisión de } \\
\text { las Comunidades Europeas }\end{array}$ & 408.296 & 113.121 \\
\hline
\end{tabular}

Fuente: elaboración propia a partir de la Base de Datos SABI

Tabla 4

Agrupación de empresas por sectores.

\begin{tabular}{|l|l}
\hline Código & Título \\
\hline & \multicolumn{1}{c}{ AGRICULTURA } \\
\hline A & AGRICULTURA, GANADERÍ, SILVICULTURA Y PESCA \\
\hline & \multicolumn{1}{|c}{ INDUSTRIA } \\
\hline B & INDUSTRIAS EXTRACTIVAS \\
\hline C & INDUSTRIA MANUFACTURERA \\
\hline D & SUMINISTRO DE ENERGIA ELECTRICA, GAS, VAPOR Y AIRE ACONDICIONADO \\
\hline E & $\begin{array}{l}\text { SUMINISTRO DE AGUA, ACTIVIDADES DE SANEAMIENTO, GESTIÓN DE } \\
\text { RESIDUOS Y DESCONTAMINACIÓN }\end{array}$ \\
\hline & \multicolumn{1}{|c}{ SERVICIOS } \\
\hline G & CONSTRUCCIÓN \\
\hline & \\
\hline F & COMERCIO AL POR MAYOR Y AL POR MENOR; REPARACIÓN DE VEHÍCULOS DE \\
\hline M & TRANSPORTE Y ALMACENAMIENTO \\
\hline I & HOSTELERÍA \\
\hline J & INFORMACIÓN Y COMUNICACIONES \\
\hline L & ACTIVIDADES INMOBILIARIAS \\
\hline M & ACTIVIDADES PROFESIONALES, CIENTÍFICAS Y TÉCNICAS \\
\hline N & ACTIVIDADES ADMINISTRATIVAS Y SERVICIOS AUXILIARES \\
\hline P & EDUCACIÓN \\
\hline Q & ACTIVIDADES SANITARIAS Y DE SERVICIOS SOCIALES \\
\hline R & ACTIVIDADES ARTÍSTICAS, RECREATIVAS Y DE ENTRETENIMIENTO \\
\hline S & OTROS SERVICIOS \\
\hline & \\
\hline K & ACTIVIDADES FINANCIERAS Y DE SEGUROS \\
\hline O & ADMINISTRACIÓN PÚBLICA Y DEFENSA; SEGURIDAD SOCIAL OBLIGATORIA \\
\hline T & $\begin{array}{l}\text { ACTIVIDADES DE LOS HOGARES COMO EMPLEADORES Y COMO PRODUCTORES } \\
\text { DE BIENES Y SERVICIOS PARA USO PROPIO }\end{array}$ \\
\hline U & ACTIVIDADES DE ORGANIZACIONES Y ORGANISMOS EXTRATERRITORIALES \\
\hline
\end{tabular}

Fuente: elaboración propia a partir de CNAE2009. 
Tabla 5

Población de empresas desagregada por sector y tamaño.

\begin{tabular}{lrrrrr}
\hline & Agricultura & Industria & construcción & \multicolumn{1}{c}{ Servicios } & \multicolumn{1}{c}{ Total } \\
\hline Pequeña & 208 & 21814 & 23.654 & 49.728 & 95.404 \\
Mediana & 258 & 4391 & 3.373 & 8.765 & 16.787 \\
Grande & 42 & 949 & 450 & 2.063 & 3.504 \\
\hline Total & 508 & 27154 & 27.477 & 60.556 & 115.695 \\
\hline
\end{tabular}

Fuente: elaboración propia a partir de la Base de Datos SABI

\section{A la búsqueda de la empresa familiar en SABI una historia en etapas}

La utilización de SABI en los trabajos de investigación españoles sobre EF es habitual. Así, Santana y Cabrera (2001:10) la utilizan para determinar si alguna familia tienen mas de un $10 \%$ de propiedad y está representada en el Consejo de Administración para lo que utilizan la coincidencia de apellidos entre sus miembros.

Por su parte, Seijo y Menéndez (2007:4) utilizan SABI para identificar, clasificar y analizar la estructura de propiedad y gestión de cada empresa, de tal forma que se consideran familiares aquellas empresas en las que existe parentesco entre el accionariado/presidente y los administradores/directivos de la empresa, ya sea parentesco de descendencia directa (padre, madre, hijos), parentesco entre hermanos o parentesco indirecto (primos, sobrinos, tíos).

En igual sentido proceden Maseda et al (2009:39), que revisan la estructura del accionariado y su porcentaje en el capital, así como la composición del órgano de administración y de gestión de la base de datos de empresas previamente seleccionada.

A pesar de este recurso habitual a SABI, la revisión de los mismos pone de manifiesto dificultades operativas en la búsqueda de la EF que no quedan suficientemente explicitadas.

La búsqueda de un proceso homogéneo de tratamiento de los datos para la clasificación de las empresas entre EFs y EnFs requiere cumplir unas etapas que describimos a continuación.

\subsection{Primera etapa: accionista físico o familia}

Resulta lógico buscar criterios automáticos en SABI que nos permitan discriminar entre EFs y EnFs. Lo primero es intentar obtener una población de empresas familiares tomando como variable de identificación el tanto por ciento de participación en manos de personas o familias, ya que este es uno de los factores cuantificables fácil de obtener.

SABI ofrece la posibilidad de búsqueda automática de datos a partir del comando "accionista una o más personas físicas o familias".

Aparentemente, dicho criterio permite discriminar las empresas por porcentaje de participación en manos de los accionistas personas físicas o familias. En teoría podemos conocer cuáles son las empresas cuyos accionistas -personas físicas o familias- poseen el $1,10,20, \ldots$ por ciento de su capital social y, por tanto, decidir que son EFs aquéllas en las que personas o familias poseen, por ejemplo, más del $50 \%$ de las acciones.

Si profundizamos en el significado del criterio advertimos que sólo funciona con 'accionistas inmediatos', aquellos que directamente poseen esa participación, dejando fuera a todas las empresas que están en manos de personas físicas o familias de forma indirecta.

Lo que realmente interesa para calificar a una empresa como familiar es \% de participación del propietario último ${ }^{5}$, sobre todo teniendo en cuenta que muchas familias ostentan el control y gestionan sus empresas a través de sociedades

\footnotetext{
${ }^{5}$ Por ejemplo, Isidoro Álvarez tiene aproximadamente el $15 \%$ de "El Corte Inglés" y el "El Corte Inglés" tiene el 100\% de varias filiales. Sin embargo, cuando aplicamos el criterio "accionista una o más personas físicas o familias" con un tanto por ciento del $10 \%$ a las empresas filiales mencionadas no aparece como accionista Isidoro Álvarez. Por lo tanto, estamos dejando fuera a empresas que realmente son familiares, pero que son participadas por sociedades interpuestas.
} 
interpuestas, por motivos fiscales o de otra índole (Sacristán y Gómez, 2007:247-248).

Santana y Cabrera (2001:10) son conscientes de este problema y utilizan SABI para determinar la propiedad de las sociedades interpuestas con el objeto de definir al último propietario en la cadena de propiedad y para determinar la composición del consejo.

En definitiva, el criterio "accionista una o más personas físicas o familias" no satisface la búsqueda del propietario último, en orden a calificar una empresa como posible familiar o no. Por tanto, cualquier investigación basada en este criterio introduce sesgos importantes que se han de evitar.

\subsection{Segunda etapa: la matriz global de SABI}

Dada la incapacidad del criterio "accionista una o más personas físicas o familias" podemos utilizar el criterio "matriz global" de SABI que, supuestamente, es capaz de conseguir el accionista o propietario final de cada empresa.

Este criterio puede ser cruzado con el \% de participación combinándolo con los indicadores de independencia que SABI suministra y a los que nos referimos en la tercera etapa.

Sin embargo, hay muchas empresas de cuyo carácter familiar nadie duda, pero para las que la base de datos no ofrece el nombre de la matriz global porque no conoce el tanto de participación del propietario final.

Se quedan así fuera demasiadas EFs para que un estudio empírico basado en él sea suficientemente fiable. Sirva como ejemplo el hecho de que para 577 de 900 empresas pequeñas del sector de la construcción tomadas aleatoriamente, SABI desconocía la matriz global y los accionistas inmediatos.

Numerosas empresas que administran su propiedad mediante sociedades patrimoniales no suelen publicar la información relativa al \% de participación de sus accionistas.

Recordemos que el Plan General de Contabilidad de 1990 y el actual solicita información sobre el capital poseído por otra empresa, directamente o por medio de sus filiales, cuando sea igual o superior al $10 \%$, pero no de las personas físicas o familias que posean un determinado $\%$ en la empresa.

\subsection{Tercera etapa: el indicador de independencia.}

Visto que el criterio matriz global es también insuficiente para la búsqueda de la población de empresas familiares, se pueden filtrar las empresas atendiendo al criterio SABI 'indicador de independencia' (II).

SABI califica las empresas atendiendo a cuatro niveles o II: A, B, C y D. El II-A significa que la empresa no depende de otra, es independiente.

Una empresa con indicador B, C ó D es una empresa dependiente de la que, alguna empresa o persona - propietario último-, tiene más del $25 \%$ de sus acciones.

Atendiendo a esta variable, las empresas que tiene indicador B, C ó D y con una matriz global 'accionista persona física o familia' podrían conformar una primera aproximación a la población de empresas familiares.

Sin embargo, se advierte que la estrategia de considerar a toda empresa con un II-A como EnF es errónea.

El II-A sólo indica que ninguna participación final -en manos de una sola empresa o personaes superior al $25 \%$, pero existen casos en que la participación en manos de la familia (hermanos, hijos,...) es superior al $25 \%$, aunque ninguno de los miembros individualmente considerados lleguen a tal porcentaje.

En definitiva, el II-A nos dice que: o bien se trata de EnF, o bien de EF con la propiedad bastante repartida (segunda o siguientes generaciones).

\subsection{Cuarta Etapa: trabajo manual.}

Parece claro que los indicadores SABI previamente analizados, que a priori parecían servirnos para detectar EFs automáticamente 
("accionista una o más personas físicas o familias", "matriz global", "indicador de independencia"), son insuficientes para una segura detección.

No asumir riesgo en la determinación de la población requiere proceder a una clasificación forzada o manual ("una a una" por sectores y tamaños) a partir de la población de empresas seleccionada.

Ya que no podemos utilizar los filtros automáticos SABI para identificar a una empresa como familiar, no hemos tenido otra opción que analizar en cada empresa los apellidos y otras características de los propietarios y de los órganos de decisión, como se verá en los párrafos posteriores.

Por ejemplo la empresa ACEDO HERMANOS S.L. es propiedad de Rafael Acedo Gorbacho y de Manuel Acedo Gorbacho, el CEO es Rafael Acedo Gorbacho y el Consejo de Administración está formado además de por las dos personas citadas anteriormente, por Concepción Acedo Olmedo y por Manuel Jesús Acedo Olmedo.

La coincidencia de apellidos entre los grupos de interés interno justificará la clasificación de la empresa entre las familiares.

Como hemos comentado y comprobado anteriormente, frecuentemente en SABI se desconoce tanto los accionistas inmediatos como los finales, por lo que no es posible clasificar las empresas atendiendo únicamente a la propiedad.

Un buen mecanismo para tratar de discernir cuando hablamos de una EF es analizar el grado de influencia decisiva sobre los aspectos fundamentales del gobierno corporativo.

Para ello no queda otra opción que analizar el grado a presencia relevante en los órganos de gobierno y administración por parte de los miembros de la familia (habitualmente el Consejo de Administración) ó mediante la participación directa de un miembro de la familia en la gestión de la empresa a su máximo nivel (CEO).
Para ello podemos comparar los apellidos de todos los grupos de interés internos implicados en la gestión y dirección del negocio: accionistas inmediatos $\mathrm{y}$ finales, CEO $\mathrm{y}$ administradores de la empresa ${ }^{6}$, lo que requiere solicitar a SABI la siguiente información por empresa: forma jurídica, accionista matriz global, accionista inmediato, CEO -director ejecutivo- y administradores.

Estudios como los de Gómez-Mejía, NuñezNickel y Gutiérrez (2001: 87) apoyan el interés de esta forma de proceder.

Este sistema 'empresa a empresa' o, como decíamos anteriormente, manual, es laborioso pero permite establecer un amplio número de categorías de EF y EnF, en función de cómo se combinan los datos anteriores. Sin embargo, una clasificación práctica y operativa obliga a:

1. reducir el número de categorías para evitar que para algunas de estas tipologías no existan casos o su número sea muy reducido.

2. desechar aquéllas empresas en las que no sea posible comprobar, con los datos disponibles, si cumplen o no la definición de EF.

En este sentido, se deben dejar fuera aquellas empresas cuyos accionistas o administradores sean a su vez empresas ó aquéllas en las que no se pueda comprobar claramente que hay coincidencia de apellidos.

Una clasificación operativa de las empresas por sectores y tamaño, a partir de la información suministrada por SABI, dadas las dificultades operativas descritas en las etapas anteriores, se muestra en el gráfico 1.

\footnotetext{
${ }^{6}$ En España resulta de gran capacidad explicativa el uso de los dos apellidos, frente a otros países dónde únicamente figura uno, ya que posibilita que hasta los apellidos más corrientes se conviertan en una coincidencia inusual si se combinan de dos en dos. Por ejemplo, quizás "Pérez" ó "López" por separado haya muchos, pero la probabilidad de ocurrencia diminuye drásticamente si se trata de encontrar "Pérez López".
} 
Gráfico 1

Definición operativa de empresa familiar en SABI.
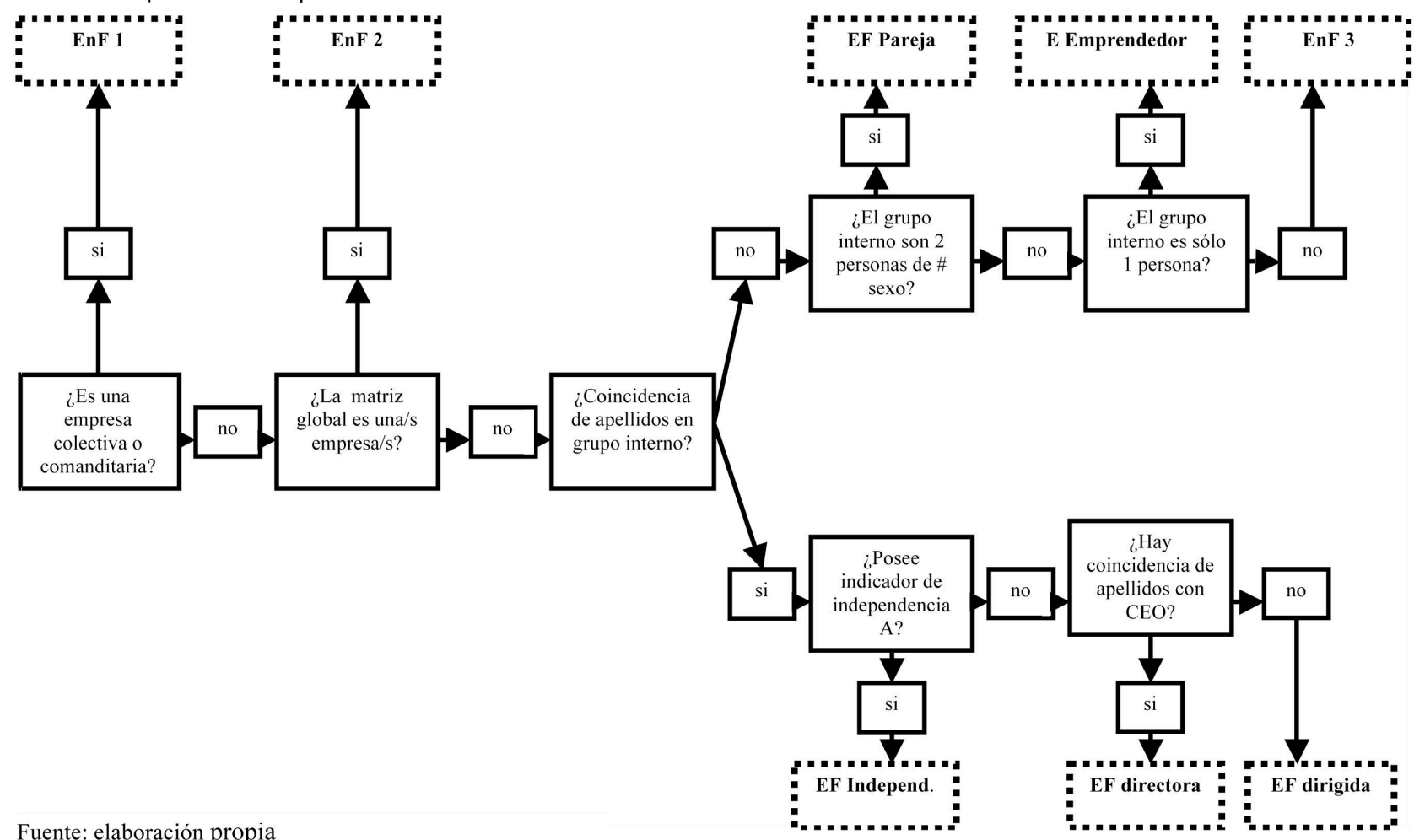

Fuente: elaboración propia

posee más del $25 \%$ del accionariado, se

Tipo 1. Sociedades colectivas $y$ comanditarias. Se supone que las empresas que han elegido como forma jurídica la colectiva o comanditaria no tienen vocación de continuidad.

Tipo 2. Hay matriz global -accionista finaly ésta es una empresa/s. Aquélla/s empresa/s cuyo accionista final o matriz global es otra empresa/s no es de naturaleza familiar. Se entiende que sólo podrán ser familiares aquéllas en las que los accionistas últimos son personas.

Tipo 3. No hay coincidencia de apellidos entre los grupos de interés internos involucrados en la gestión y dirección de la empresa. Si no hay coincidencia clara de apellidos en los grupos descritos como accionistas, CEO y administradores, no existen lazos familiares ni matrimoniales entre ellos, y por tanto, la empresa no es de carácter familiar.

Tipo 4. EF independiente. Hay coincidencia de apellidos entre los grupos de interés internos reseñados y el 'II' es "A" (II-A). Como el indicador " $A$ " otorgado por SABI designa a aquellas empresas en las que ningún accionista considera a este tipo como EF, en tanto existen lazos familiares no matrimoniales entre los grupos de interés internos señalados, pero la propiedad está muy repartida.

Tipo 5. EF dirigida. Hay coincidencia de apellidos entre accionistas y/o administradores, por lo que existen lazos familiares no matrimoniales entre los mismos, y esos apellidos no coinciden con los del CEO. En este apartado se clasifican las empresas familiares con dirección profesionalizada. Alguien externo a la familia dirige la sociedad.

Tipo 6. EF directora. Hay coincidencia de apellidos en accionistas y/o administradores, y esos apellidos coinciden con los del CEO. En este caso, la familia ha encargado a uno de sus miembros la dirección de la empresa.

Tipo 7. Empresas pareja. Muy similares a la anterior formalmente, pero en accionistas y/o administradores y/o CEO sólo hay 2 personas de distinto sexo y con diferentes apellidos, por lo que suponemos existen lazos familiares matrimoniales entre los mismos. 
Tipo 8. Empresas emprendedor. Suele haber una sola persona que es todo (accionista, CEO y administrador), y no es criterio "A"; la matriz global es esa misma persona, y se ve que normalmente detenta un $\%$ mayor que el $50 \%$. Recae en la misma persona la condición de accionista, administrador y CEO.

Volviendo a la clasificación propuesta $\mathrm{y}$ según los pasos indicados previamente, las empresas pueden quedar clasificadas como sigue:

1. Los tipos 1 a 3 son, con toda probabilidad, empresas no familiares;

2. Los tipos 4 a 7 son, con toda probabilidad, empresas familiares;

3. El tipo 8, sería el origen de toda empresa familiar, el emprendedor. Realmente no podemos considerarlo como empresa familiar, aunque hay estudios que así lo consideran (Astrachan y Schanker, 2003: 214), por ejemplo, en el caso de las granjas.

\section{Hacia una conciliación de los conceptos de empresa familiar}

Tal como se dijo en el apartado 2, existe cierta disociación entre el concepto teórico y el operativo de EF. La aplicación de una u otra definición da lugar a interpretaciones de la realidad muy diferentes, tal como ponen claramente de manifiesto (Astrachan y Schanker, 2003) en su estudio sobre el papel de la EF en la economía de EE. UU.

Este trabajo realiza un estudio del concepto de EF desde la perspectiva operativa, antes que teórica, a sabiendas de que la primera queda más limitada al plano de la investigación empírica, pero conscientes de la importancia que ello tiene para avanzar en el estudio de la $\mathrm{EF}$.

La búsqueda de una definición operativa sustentada en SABI nos permite en el Camino descubrir una tipología de EF que puede resultar de interés para la investigación facilitando estudios en función del tipo de empresa que analizamos.

No obstante, esta definición operativa mantiene un vacío casi insalvable con el concepto teórico: la apreciación del deseo de transmisión o el deseo de pertenencia, aspectos estos de naturaleza subjetiva e ideológica que resulta enormemente difícil de apreciar en la frialdad de los datos objetivos.

A nuestro juicio, el esfuerzo por clasificar una empresa como familiar o no, sobre la base de SABI, ayuda a una mejor interpretación de los datos resultantes de los trabajos de investigación, lo que constituye un importante paso, pero el aspecto relativo al sentimiento de pertenencia difícilmente puede ser aprehendido por medio de dicha base.

El acercamiento de las definiciones operativas y teóricas, al menos por el momento, sólo puede venir de la mano de la pregunta directa al interesado: 1) ¿consideras que tu empresa es familiar?; 2) ¿existe el deseo de transmitirla a las siguientes generaciones?, siendo esta segunda cuestión clave para las empresas catalogadas en este trabajo dentro del tipo 8 .

El investigador habrá de decidir, en base al objetivo perseguido, hasta donde llegar, lo que probablemente le obligue, en algunas ocasiones, a dividir su investigación en etapas.

\section{Conclusiones}

Aunque existe cierto consenso en relación con el concepto de EF, al tratarse de un concepto multifactorial y multidisciplinar, no está exento de discusión su tratamiento. En el ámbito investigador resulta útil la diferencia entre un concepto teórico, orientado a crear teoría, y otro operativo, imprescindible para clasificar a una empresa como familiar o no.

No obstante, no resulta fácil conciliar uno y otro, debido a la existencia de componentes subjetivos como son el de pertenencia o el deseo de transmisión a las generaciones futuras, por lo que el tipo de investigación y el objetivo perseguido mostrarán al investigador el camino a seguir. En cualquier caso queda aquí una línea de trabajo abierta que es necesario explorar y profundizar.

La diferencia entre EF y EnF en España, en el ámbito operativo, suele hacerse con la base de datos SABI. Nuestro trabajo muestra que los 
criterios de automatización de dicha base: "accionista una o más personas físicas o familias", "matriz global" e "indicador de independencia", resultan en gran medida infructuosos para tal discriminación, ya que no siempre se conoce quiénes son los accionistas finales de las empresas y, sin embargo, es muy habitual que las familias ostenten el control y gestionen sus empresas a través de sociedades interpuestas.

El uso de dos apellidos en España justifica la búsqueda del carácter familiar o no de las empresas a través de la comparación de apellidos de los accionistas, CEO y administradores de la empresa.

De esta forma la diferenciación entre EF y EnF, se puede sustentar, no sólo en función de quién posea las acciones, también en función de quien ejerza la influencia decisiva sobre los aspectos fundamentales del gobierno corporativo, a través de una presencia relevante en los órganos de gobierno corporativo de la compañía (habitualmente el Consejo de Administración) ó mediante la participación directa de un miembro de la familia en la gestión de la empresa a su máximo nivel (CEO).

Finalmente, el sistema de búsqueda utilizado permite establecer una tipología de empresas familiares que puede resultar de interés para futuros trabajos de investigación.

En particular, la categoría tipo 8 es la más controvertida por dos motivos: 1) porque en ella se recoge a los emprendedores y empresas 'personalistas'; 2) porque es en estos casos en los que es más necesario conocer la opinión del 'empresario' y más difícil llegar a él; 3) porque a menudo se le presta menor atención debido a su menor peso en el PIB.

Señalar que este trabajo abre un camino que pasa forzosamente por contrastar los resultados de su adopción en dos sentidos: a) realizando estudios empíricos sobre las muestras obtenidas y comparando con estudios previos no concluyentes; b) analizando hasta que punto los diferentes tipos de EF adoptan comportamientos distintos a la luz de las teorías existentes.

\section{Bibliografía}

Allouche, J., Amann, B., Jaussaud, J., y Kurashina, T. (2008). The Impact of Family Control on the Performance and Financial Characteristics of Family Versus Nonfamily Businesses in Japan: A Matched-Pair Investigation. Family Business Review, 21(4), 315-329.

Anderson, R.C. y Reeb, D. M. (2003). Founding-Family Ownership and Firm Performance: Evidence from the S\&P 500. The Journal of Finance, 58(3), 1301-1329

Ariza, J.A., Molina, H. y Ramírez, J. (2005). El concepto de empresa familiar. XIII Congreso de AECA. Oviedo. Septiembre.

Astrachan, J.H. y Shanker, M.C. (2003). Family Businesses Contribution to the US Economy: A Closer Look. Family Business Review, 16(3), 211-216.

Basco, J.T. (2006). La Investigación En La Empresa Familiar: Un Debate Sobre La Existencia De Un Campo Independiente. Investigaciones Europeas en Dirección y Economía de la Empresa, 12(1), 33-54.

Chrisman, J.J., Chua, J. H. y Steier, L. P. (2005): Sources and Consequences of Distinctive Familiness: An Introduction. Entrepreneurship Theory and Practice, 29(3), 237.

Chrisman, J.J., Chua, J. H. y Sharma, P. (2005). Trends and Directions in the Development of a Strategic Management Theory of the Family Firm. Entrepreneurship Theory and Practice, 29(5), 555-575.

Chrisman, J.J., Chua, J. H. y Litz, R. (2003). A Unified Systems Perspective of Family Firm Performance: An Extension and Integration. Journal of Business Venturing, 18(4) 467

Chua, J.H., Chrisman, J. J. y Sharma, P. (1999). Defining the Family Business by Behavior. Entrepreneurship Theory and Practice, 23(4), 19.

Comblé, K. et Colot, O. (2006). L'entreprise Familiale: Concept Et Importance En Belgique. Reflets Et Perspectives. XLV(2), 91-108. 
Dirección General de Política de la Pequeña y Mediana Empresa (2008). Guía para La Pequeña y Mediana Empresa Familiar. $\left(6^{\mathrm{a}}\right.$ edición). Madrid: Ministerio de Industria, Turismo y Comercio.

Dyer, W.G., Jr (2006). Examining the "Family Effect" on Firm Performance. Family Business Review, 19(4), 253-273.

Gallo, M.A, Tápies, J. y Kappuyns, K. (2004). Comparison of Family and Nonfamily Business: Financial Logic and Personal Preferentes. Family Business Review, 4(4), 303.

Galve, C. y Salas, V. (1996). Ownership Structure and Firm Performance: Some Empirical Evidence from Spain. Managerial and Decision Economics (1986-1998), 17(6), 575.

García de la Borbolla, A., Herrera, J., Larrán, M, Sánchez, G. y Suárez, A. (2007). Análisis Empírico de las Diferencias de Eficiencia entre las Empresas Familiares y las no Familiares. Investigaciones Europeas en Dirección $y$ Economía de la Empresa, 13(1), 103-116.

Garcia de la Borbolla, A., Herrera, J., Larrán, M, Sánchez, G. y Suárez, A. (2006). Factores de éxito de la Empresa Familiar. Estudio aplicado a la Empresa Andaluza. Madrid: AECA

Gómez-Mejía, L.R., Nuñez-Nickel, M. y Gutiérrez, Isabel (2001). The role of family ties in agency contracts. The Academy of Management Journal, 44 (1), 81-95.

Habbershon, T.G., Williams, M. y MacMillan, I. C. (2003). A Unified Systems Perspective of Family Firm Performance. Journal of Business Venturing, 18(4), 451.

Handler, W.C. (1989). Methodological issues and considerations in studying family businesses. Family Business Review, 2(3), 257276.

Heck, R.K.Z. y Scannell, E. (1999). The Prevalence of Family Business from a Household Sample. Family Business Review, 3, 209-219.
Lee, J. (2006). Family Firm Performance: Further Evidence. Family Business Review, 19(2), 103-114.

Litz, R.A. (1995). The Family Business: Toward Definitional Clarity. Family Business Review, 8(2), 71-81.

López-Gracia, J. y Sánchez-Andújar, S. (2007). Financial Structure of the Family Business: Evidence from a Group of Small Spanish Firms. Family Business Review, 20(4), 269-287.

McConaughy, D. L., Matthew, C.H. y Fialko, A.S. (1998). Founding Family Controlled Firms: Efficiency and Value. Review of Financial Economics, 7(1), 1-19.

Mandl, I. (2008). Overview of Family Business Relevant Issues. Final Report. European Commission, Enterprise and Industry Directorate-General. Vienna.

Martinez, J., Stohr, B.S y Quiroga, B.F. (2007). Family Ownership and Firm Performance: Evidence From Public Companies in Chile, Family Business Review, 20(2). 83-94.

Maseda, A., Iturralde, T. y Arosa, B. (2009): Situación de la empresa familiar en el territorio histórico de Bizkaia. XXIII Congreso Nacional de AEDEM. Sevilla. Junio.

Monreal, J., Sánchez, G., Meroño, A.L.S. y Sabater, R. (2009). La gestión de las empresas familiares: un análisis integral. Navarra: Ed. Aranzadi. Cívitas-Thomson Reuters.

Rodríguez, I. (2006). La empresa familiar en el ámbito del Derecho Mercantil. Madrid: Ed. Edersa.

Sacristán, M y Gómez, S. (2007). Family Ownership and Pyramids in the Spanish Market, Family Business Review, 20(3). 247-265.

Sánchez, G., Larrán, M., García Borbolla, A. y Herrera, J. (2008). Influencia de la propiedad familiar sobre la eficiencia de las PYME: un análisis de diferencias basado en un concepto multicriterio de rentabilidad. Revista Internacional de la pequeña y la mediana empresa, 1(2). 42-63.

Santana, D. y Cabrera, K. (2001). Comportamiento y resultados de las empresas 
cotizadas familiares versus no familiares. XI Congreso Nacional de ACEDE. Zaragoza.

Seijo, E y Menéndez, S. (2007). Eficiencia de las empresas familiares en España: Importancia de las estructuras de propiedad y control. Actas del XIX Congreso Anual de AEDEM y XV Congreso Hispano Francés de AEDEM. VitoriaGazteiz.

Sharma, P. (2004). An Overview of the Field of Family Business Studies: Current Status and Directions for the Future. Family Business Review, 17(1). 1-36.

Smith, M. (2007). Real Managerial Differences between Family and Non-Family Firms. International Journal of Entrepreneurial Behaviour \& Research, 13(5), 278.
Villalonga, B. y Amit, R. (2006). How do Family Ownership, Control and Management Affect Firm Value? Journal of Financial Economics, 80(2), 385-417.

Westhead, P. y Howorth, C. (2007). 'Types' of Private Family Firms: An Exploratory Conceptual and Empirical Analysis. Entrepreneurship and Regional Development. 19(5), 405.

Westhead, P. y Cowling, M. (1998). Family Firm Research: The Need for a Methodological Rethink. Entrepreneurship Theory and Practice. 23(1), 31 .

\begin{tabular}{|c|c|c|c|c|c|c|}
\hline \&. Santander & INStTUTO DE LA I I FMPRES & (n) & Empresa Familiar & $\frac{2}{2}$ & 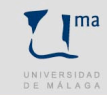 & tin \\
\hline
\end{tabular}

